\title{
Research on the robust optimization of the enterprise's decision on the investment to the collaborative innovation: Under the risk constraints is
}

\author{
Qing Zhou, Gang Fang, Dongpeng Wang, Wei Yang* \\ School of Management, Hangzhou Dianzi University, Hangzhou, China
}

\begin{abstract}
The robust optimization model is applied to analyze the enterprise's decision of the investment portfolio for the collaborative innovation under the risk constraints. Through the mathematical model deduction and the simulation analysis, the research result shows that the enterprise's investment to the collaborative innovation has relatively obvious robust effect. As for the collaborative innovation, the return from the investment coexists with the risk of it. Under the risk constraints, the robust optimization method could solve the minimum risk as well as the proportion of each investment scheme in the portfolio on the condition of different target returns from the investment. On the basis of the result, the enterprise could balance between the investment return and risk and make optimal decision on the investment scheme.
\end{abstract}

Keywords: Risk constraints, Collaborative innovation, Robust optimization, Game

\section{Introduction}

Currently, the "innovation-driven development" has become a significant strategy for China to accelerate the transformation of the economic development pattern. According to the report to the 18th National Congress of the Communist Party of China and 2015 Chinese government work report, China will "place greater emphasis on making innovation through collaboration, and establish a system of technological innovation in which enterprises play the leading role, the market points the way, and enterprises, universities as well as research institutes work together". These statements indicate that the collaborative innovation is the key way for the implementation of innovation-driven development strategy. Researchers in China have different opinions on the collaborative innovation in China. Chen and Yang [1] believed that the core of the collaborative innovation is increasing the human knowledge, and the collaborative innovation is essentially an organizational model for enterprises, government, knowledge production sectors (such as universities, research institutions), intermediaries and customers collaborate extensively for major S \& T innovation in quite a wide range of fields. Hong [2] argued that the China's version collaborative innovation is not a general sense of collaborative innovation between peers or members of a supply chain, neither be a simple inheritance of the university-industry cooperation. Instead, it is a system in which universities, enterprises, S \& T intermediaries and financing institutions conduct joint operations. So, Zeng et al. [3] concluded that the collaborative innovation should be an ecosystem

\footnotetext{
* Corresponding author

Email address: young7810@163.com (Wei Yang )
} 
in which the participants integrate with each other, creat new value chains and then generate an open network of collaboration. The participants and their resources constitute a structure of mutualistic symbiosis in this ecosystem, and the ecosystem would optimize for the best of all the participants through its evolution. During the process of the collaborative innovation, how the enterprise achieves the goal of benefit maximization through knowledge transfer and S \& T achievements transformation between each other is the key to the continuous development of the collaborative innovation.

But in fact, the collaborative innovation is faced by huge risks and challenges, especially the performance of collaborative innovation would be directly influenced by the participants' game behaviors. In order to analyze the participants' decision making behaviors in the collaborative innovation, many researchers construct the game analysis model. Zhou et al. [4] developed a dynamic model of cooperation R \& D network-based game and analyze firms' R \& D investment decisions by integrating independent research and cooperative research. The research reveals that a firm's investment in the independent projects as well as in each of the joint projects increases with collaborative degree and exogenous coefficient, and decreases with collaborative size. Qi et al. [5] presented a game model among government, enterprises, as well as university and institution. Xu et al. [6] analyzed the collaborative relationship between enterprises based on the "prisoner dilemma" model, and pointed out how enterprises maximally enhance the performance of the collaborative innovation. Then, Xu et al. [6] discussed how the enterprises and government optimize their own benefit through evolutionary game in the process of collaboration. Based on the game rules concluded by their research, the measures to realize the collaborative innovation has been proposed. Liu et al. [7] constructed a profit distribution model of universityindustry collaborative innovation value chain based on double efforts by utilizing the thought and methodology of the game theory and conflict economics. Then, the optimization model was used to solve the optimal effort degree and the optimal cooperation degree with non-coordination and coordination. Huang et al. [8] proposed a game model of collaborative innovation with venture capital funding. Their research found that the fund should give the whole revenue share ratio to the enterprise, while the fund and the innovation supplier should claim a fixed revenue from enterprise to motivate the enterprise to make commitment according to contract. The fund should give the supplier the investment later during the period of innovation, while the innovation supplier invests its own capital at earlier stages, in order to stimulate it to raise innovation commitment. As a result, the expected profit of the fund is maximized. Arsenyan et al. [9] proposed a model integrating trust, coordination, co-learning, and co-innovation dimensions of collaborative research between firms, and the analysis investigated the effect of various parameters on the collaboration formation as well as the revenue sharing under various scenarios with the Nash Bargaining theory, and finally summarized the optimum strategies for each scenario. To sum up, most researches have considered the profit distribution problem between participants of the collaborative innovation in stable environments. Few scholars conduct the research about the collaborative innovation in dynamic environments. However, the risk factors in dynamic environments should be paid more attention by the participants in the collaboration.

In dynamic environments, enterprises have to take the external environments into account when they make the investment decision, and especially the competing environment(D'Aveni et al. [10], Porter [11]). Drazin and 
Van de Ven [12] believe that the goal of survival and development make enterprises to keep the internal decisionmaking matched with external environments. As for the collaborative innovation, the uncertainty of external environments has remarkable influences to the innovation performance, so the R \& D investment decision of each enterprise should match the dynamic environments (Choi [13], Katsoulacos and Ulph [14], Narula [15]). Some literatures constructed the analytical framework for Synergetic Innovation (SI) based on Synergetics to characteristics and evolution mechanism of SI of emerging industriesshowing that SI of emerging industries was a complex process of the interaction between the internal driving of innovation subject of industrial innovation system and external environmentand SIis in the dynamic process of evolution with the development of the industrieswhich was from breeding phase to germination phase and growth phase (Chen and Sui, [16]). The current researches about investment decision of collaborative innovation have not considered the risks and benefits of it, and the uncertainty of dynamic environments has not been analyzed. Therefore, in this paper we study the enterprises' decision on the investment to collaborative innovation under the risk constraints in the uncertainty environment. An analysis is conducted with the robust optimization model to investigate the impact of dynamic environments, solve the minimal risk of the portfolio and provide an investment plan for the collaborative innovation.

\section{Collaborative innovation decision analyses under the dynamic environment}

According to Goyal et al. [17], enterprise collaborative innovation activities generally include three stages: (1) formation of collaborative innovation network; (2) every enterprise allocates resource to the independent and collaborative innovation activities and these decisions decide the effective cost of production; (3) the enterprises take part in the Cournot competition in product market. There are different decision problem at different stage for enterprise. Enterprises need to decide whether to participate in collaborative innovation firstly. Then, if they take part in collaborative, they should make investment decision. At last, according to the result of innovation, enterprises would consider the decision of competition. Based on above, we can build the model as follows.

\subsection{Collaborative innovation decision analyses}

Suppose $N=\{1, \cdots, n\}(n \geq 2)$ is a set of enterprises on the market. The collaborative networks formed in the first stage can be expressed by $g$. If $i \in g$, it means that the company $i$ participates in the collaborative network. $N_{i}(g)$ denotes the enterprises set which has collaborative relationship with enterprise $i$ in network g. $n_{i}(g)=\left|N_{i}(g)\right|$ is the number of collaborative enterprises with enterprise $i$ in network $g$. In this paper, we define it as the collaborative innovation scale of enterprise, denoted by $k \in\{1,2, \cdots, n\}, k=1$ means completely independent innovation and $k>1$ means the formation of collaborative innovation network.

Suppose $g$ is the network generated in the first stage, and $x_{i g}$ is the collaborative innovation investment of enterprise $i$ in $g$. To maintain the core competition ability, enterprise invests $x_{i i}$ for improving independent innovation. $x=\left(x_{i g}, x_{i i}\right)$ is the strategic choice in the game of enterprise $i$ and $x=\left(x_{i}\right)_{i \in N}$ represents the strategy portfolio in the game. 


\subsection{Investment decisions portfolio of collaborative innovation}

Suppose $f(X)\left(f^{\prime}(X)>0, f^{\prime \prime}(X)<0\right)$ is the production function of innovation investment, where $X$ is the effective innovation investment, i. e., the required investment to achieve the same range of cost reduction when other companies do not make R \& D investment. The production function of innovation investment for enterprise $i$ can be described by

$$
f_{i i}(x)=f\left(x_{i i}\right)
$$

In collaborative innovation, the effective investment of collaborative innovation and the production function are given respectively as follows

$$
X_{i g}=\sum_{i \in g} x_{i g}, f_{i g}(x)=f\left(X_{i g}\right)
$$

The reduction of production cost caused by carrying out innovation activities can be represented by

$$
f_{i}(x)=f\left(X_{i g}\right)+f\left(x_{i i}\right)
$$

So in the second stage the unit production cost of the enterprise is

$$
c_{i}(g, x)=\bar{c}-f_{i}(x)
$$

where $\bar{c}$ stands for the unit production cost of the enterprise before carrying out innovation activities.

\subsection{The benefits of enterprises to participate in Cournot competition in product market}

Taking the cost $c_{i}(g, x)$ into account, enterprise $i$ chooses a certain number of $\left(q_{i}(g, x)\right)_{i \in N}$ to participate in Cournot competition in product market. Suppose the product demand inverse function of enterprise $i$ is symmetric linear, which is

$$
p=a-q_{i}-\varepsilon \sum_{j \neq i} q_{j}(g, x)
$$

where $\varepsilon \in[0,1]$ is the product substitution coefficient. In over-supply of the homogeneous product markets, the equilibrium quantity for enterprise $i$ can be generated as follows(Goyal and Joshi [17], Bala and Goyal [18], Kranton and Minehart [19]):

$$
q_{i}(g, x)=\frac{(2-\varepsilon) a-(n \varepsilon-2 \varepsilon+2) c_{i}(g, x)+\varepsilon \sum_{j \neq i} c_{j}(g, x)}{(\varepsilon n-\varepsilon+2)(2-\varepsilon)} .
$$

The equilibrium profit can be expressed as

$$
\pi_{i}(g, x)=\left(q_{i}(g, x)\right)^{2}-\sum_{j \in \mathrm{N}_{i}(g)} x_{i j}-x_{i i} .
$$

\subsection{The investment equilibrium outcomes under the maximum profit of enterprise}

Taking into account network $g$ and other innovation investment of enterprise $i, \pi_{i}(g, x)$ is maximized at $x_{i}$ with $x_{i i} \geq 0, x_{i j} \geq 0, j \in N_{i}(g)$. The research problem can be described as follows

$$
\begin{array}{ll}
\max & \pi_{i}(g, x) \\
\text { s.t. } & x_{i i} \geq 0, \\
& x_{i j} \geq 0, j \in N_{i}(g) .
\end{array}
$$


The first-order conditions for maximizing the above problem are given as follows:

$$
\begin{gathered}
\frac{\partial \pi_{i}}{\partial x_{i i}}(x)=\frac{2 q_{i}(g, x)(n \varepsilon-2 \varepsilon+2) f^{\prime}\left(x_{i i}\right)}{(\varepsilon n-\varepsilon+2)(2-\varepsilon)}-1=0, \\
\frac{\partial \pi_{i}}{\partial x_{i g}}(x)=\frac{2 q_{i}(g, x)(n \varepsilon-k \varepsilon-\varepsilon+2) f^{\prime}\left(R_{i g}\right)}{(\varepsilon n-\varepsilon+2)(2-\varepsilon)}-1=0 .
\end{gathered}
$$

In order to get solution of the above optimization problem, we can reference the research conducted by Goyal and Moraga-Gonzalez [20], Goyal and Moraga-Gonzalez [21]. Suppose $f(X)=(1 / \gamma) \sqrt{X}$ and $g=g^{k}$ is a symmetry network of collaboration scale $k$, of which $\gamma \in(0,+\infty)$ represents enterprise's income coefficient of innovation investment in dynamic environment. Plug $f(X)=(1 / \gamma) \sqrt{X}$ into equation (11), we get the following equation

$$
x_{i g}^{*}=\frac{(\varepsilon n-\varepsilon k-\varepsilon+2)^{2}}{k(\varepsilon n-2 \varepsilon+2)^{2}} x_{i i}^{*} .
$$

Plug the above formula into equation (9), we get the investment level under the symmetry equilibrium condition the as follows

$$
\begin{gathered}
x_{i i}^{*}=\left[\frac{\gamma(\varepsilon n-2 \varepsilon+2)[a-\bar{c}]}{\gamma^{2}(\varepsilon n-\varepsilon+2)^{2}(2-\varepsilon)-(\varepsilon n-2 \varepsilon+2)-(\varepsilon n-k \varepsilon-\varepsilon+2)}\right]^{2}, \\
x_{i g}^{*}=\frac{1}{k}\left[\frac{\gamma(\varepsilon n-k \varepsilon-\varepsilon+2)[a-\bar{c}]}{\gamma^{2}(\varepsilon n-\varepsilon+2)^{2}(2-\varepsilon)-(\varepsilon n-2 \varepsilon+2)-(\varepsilon n-k \varepsilon-\varepsilon+2)}\right]^{2} .
\end{gathered}
$$

From formula (6), (7), (11) and (12), we get the investment returns under the condition of equilibrium as follows:

$$
\pi_{i}\left(x^{*}, g^{k}\right)=\frac{[a-\bar{c}]^{2} \gamma^{2}\left[k \gamma^{2}(2-\varepsilon)^{2}(\varepsilon n-\varepsilon+2)^{2}-k(\varepsilon n-2 \varepsilon+2)^{2}-(\varepsilon n-\varepsilon k-2 \varepsilon+2)^{2}\right]}{k\left[\gamma^{2}(\varepsilon n-\varepsilon+2)^{2}(2-\varepsilon)-(\varepsilon n-2 \varepsilon+2)-(\varepsilon n-k \varepsilon-\varepsilon+2)\right]^{2}} .
$$

\section{The robust optimization model of enterprise's investment to the collaborative innovation with the risk constraints}

\subsection{The robust optimization model building}

Since the second half of the 20th century, optimization techniques had been widely applied in the fields of production planning, positioning and transport, resource allocation in the economic system and project design. Meanwhile, the problem of design and optimization under uncertainty had obtained more attention from researchers [22]. The robustness of the robust optimization solution means that the optimal solution is insensitive to uncertain data. In order to solve the mathematical programming problem with uncertain information, Soyster [23], Mulvey [24] and Bertsimas [25] have proposed the concept of robust optimization by introduce the parameters to control the balance between the violating probability of the constraints and its impact on the objective function.Considering the impact of the enterprises investment in collaborative innovation to the innovative returns under circumstances of dynamic environment, we bring in the robust optimization model and the relative thoughts to analyze the enterprise's decision on the investment portfolios about collaborative innovation. The robust optimization model is nonlinear, and is more suitable for dynamic decision problem than linear model. 
Based on the equilibrium analysis about profit maximization of the investment of collaborative innovation in the above section, let there are $\tau$ investment schemes for collaborative innovation, and the rate of return of the $i$ th scheme is $\pi_{i}$ under the equilibrium condition. Suppose the investment in each scheme is random, the weight of the $i$ th scheme is $w_{i}\left(i=1,2, \cdots, \tau\right.$ with $\left.w_{i} \geq 0, \sum_{i=1}^{\tau} w_{i}=1\right)$ which is a set conform to the constraints of linear matrix inequalities, and the covariance matrix between the $i$ th scheme and the $j$ th scheme is $B_{n \times n}$.

$$
\Gamma=\left|w \in s^{\tau}, F_{0}+\sum_{i=1}^{\tau} w_{i} F_{i} \geq 0\right| .
$$

$F_{i}(i=1,2, \cdots, \tau)$ is a known symmetric matrix. $w$ has to meet the conditions $w^{T} I \leq 1(I$ is unit column vector, $\left.w_{i} \geq 0, i=1,2, \cdots, \tau\right)$. When the enterprise's target return $E(\pi)$ is given, the robust optimization model of collaborative innovation is:

$$
\begin{gathered}
\min _{w} \chi^{2}=w^{T} B w \\
\text { s.t. } w^{T} \Pi \geq E(\pi), \\
\sum_{i=1}^{\tau} w_{i}=1, \\
0 \leq w_{i} \leq 1, i=1,2, \cdots, \tau .
\end{gathered}
$$

In the model, $\Pi=\left(\pi_{1}, \pi_{2}, \cdots, \pi_{\tau}\right)$ represents the expected returns of investment schemes, and $w=$ $\left(w_{1}, w_{2}, \cdots, w_{\tau}\right)^{T}$ contains the weight of each investment scheme. $B=\left(\chi_{i j}\right)_{\tau \times \tau}$ is a covariance matrix for $\tau$ investment schemes, $\chi$ means the maximum variance.

The objective function of the model is (15), which shows that the enterprises want to minimize the risk of the investment portfolio when the expected return is achieved. (16) consist of the constraints of expected returns from investment, and (17) and (18) are the sum and nonnegative constraints of $w$ respectively. Under uncertain market circumstance, the substitution of every product is unknown. So the returns from investment to the collaborative innovation would change as well.

The linear matrix inequality is one of the significant methods to solve the robust optimization model (Costa [26]). Based on the researches of the Costa's portfolio optimization model [26] and Gao's loan portfolio robust optimization model [27], we propose our method to solve the robust optimization model of the enterprise's decision on the investment to the collaborative innovation. Hence, (15)-(18) could convert to a model in terms of linear matrix inequality as follows:

$$
\begin{gathered}
\min _{w} \Psi \\
\left|\begin{array}{cc}
\Psi & w^{T} B_{\rho} \\
B_{\rho} w & B_{\rho}
\end{array}\right| \geq 0, \\
w^{T} \Pi \geq E(\pi), \rho=1,2, \cdots, m, \\
\sum_{i=1}^{\tau} w_{i}=1, \\
0 \leq w_{i} \leq 1, i=1,2, \cdots, \tau .
\end{gathered}
$$


In the above models, $w$ and $\chi$ are two decision variables. $w$ is a vector which represents the investment proportion of different schemes in the enterprise's portfolio. $\chi$ means the maximum variance. The objective function is $\Psi=\max _{k} \chi_{B}^{2}=\max w^{T} B_{\rho} w, \rho=1,2, \cdots, m$, which aims at finding the $w$ of the minimum risk. The meaning of $\min _{w} \Psi$ is that in the dynamic environment (i.e., $\Psi=\max _{k} \chi_{B}^{2}$ ) the enterprise would choose the $w$ which make the risk of enterprise's portfolio minimum when the investment return had achieved the target return $E(\pi)(E(\pi)$ is the enterprise's expected return from collaborative innovation).

The variables $i$ and $\rho$ are the subscript variables of the model. $i(i=1,2, \cdots, \tau)$ is the sequence number of the investment scheme. In the investment equilibrium model, the coefficient of enterprise's investment return would determine the expected return of different investment scheme. $\rho(\rho=1,2, \cdots, m)$ denotes the possible change of future market. In the dynamic environment, the enterprise's investment returns from collaborative innovation directly related to the level of competition between enterprises' products. Therefore, the substitution rate of products could be used to illustrate the dynamic degree of enterprise's environment, i. e., the higher substitution rate facing the enterprise's products, the higher dynamic degree of environment the enterprise is confronted with. The constraints $\Psi_{\rho}-w^{T} B_{\rho} w \geq 0$ are transformed into (20) according to the "Schur complement", which means the maximum variance of the return from enterprise's investment to collaborative innovation in the dynamic environment. (21) represent the constraint condition of expected return of the enterprise's investment portfolio. (22) and (23) are the sum and nonnegative constraints of $w$ respectively.

\subsection{Computation steps of the model}

Step 1 Analyze the enterprises expected returns in the dynamic environment. A set of the enterprise's expected returns can be solved according to the equation of enterprise's investment to the collaborative innovation under the equilibrium condition with the change of substitution rate of products.

Step 2 Make a prediction about the enterprise's returns from different investment scheme. The expected returns $\pi_{i}$ can be calculated from the historical data of the coefficient of enterprise's investment. Then, the corresponding covariance matrices between different investment schemes are generated.

Step 3 Apply the thought of robust optimization to build the robust optimization model of enterprise's decision on the investment to the collaborative innovation based on the above analysis.

Step 4 Set the target return of the enterprise's investment to the collaborative innovation, and then solve the maximum variance of the return from enterprise's investment to collaborative innovation in the dynamic environment under the constraints of target return by utilizing the MATLAB's LMI Toolbox.

Step 5 Figure out the robust answer $w$ with the minimum risk of investment portfolio under the constraints in dynamic environment by using MATLAB Toolbox, and then give the investment plan based on the portfolio.

\section{Numerical analysis}

Let $n=12, a=100, \bar{c}=50, k=5$. The income coefficients of innovation investment $\gamma$ for different investment programs are $2,5,10,20,40$ and 60 respectively. The product substitution coefficients $\varepsilon$ for 
different market environments are $0,0.2,0.4,0.6,0.8$ and 1.0 respectively. The beneficial matrix $R$ can be calculated by using the equilibrium benefit $\pi_{i}$ model as follows

$$
R=\left[\begin{array}{cccccc}
755.1 & 643 & 629.4 & 626.1 & 625.27 & 625.12 \\
146.9 & 142.5 & 141.92 & 141.81 & 141.74 & 141.73 \\
60 & 60.89 & 61 & 61.03 & 61.033 & 61.034 \\
31.84 & 33.51 & 33.73 & 33.78 & 33.798 & 33.8 \\
19.15 & 21.09 & 21.348 & 21.41 & 21.428 & 21.431 \\
12.13 & 14.39 & 14.693 & 14.77 & 14.787 & 14.79
\end{array}\right]
$$

Let $i$ be the rows of the matrix, $j$ be the columns of the matrix and $R_{i j}$ be the $i$ th row and $j$ th column element of the matrix. The columns represent the earnings rates under different market environments for the same kind of collaborative innovation scheme, and $R_{i j}(j=1,2,3,4,5,6)$ represent the investment returns of collaborative innovation when income coefficients of innovation investment $\gamma=2, \gamma=5, \gamma=10, \gamma=20$, $\gamma=40, \gamma=60$. The rows represent the earnings rates under the same market environment for different collaborative innovation schemes, and $R_{i j}(i=1,2,3,4,5,6)$ represent the investment returns of collaborative innovation when $\varepsilon=0, \varepsilon=0.2, \varepsilon=0.4, \varepsilon=0.6, \varepsilon=0.8$ and $\varepsilon=1.0 . \varepsilon=0$ and $\varepsilon=1.0$ are two kinds of extreme market conditions. $\varepsilon=0$ means that enterprises are in a completely monopoly market and the product substitution coefficient is zero. $\varepsilon=1.0$ means that enterprises are in a perfectly competitive market and the product substitution coefficient is one.

The expected return of different investment programs can be calculated by matrix $R$. With the weights of investment programs under different market environments $0.1,0.15,0.25,0.25,0.15$ and 0.1 , the expected return, denoted by $H$, can be calculated

$$
H=\left[\begin{array}{llllll}
124.6 & 113.9 & 112.58 & 112.3 & 112.19 & 112.17
\end{array}\right]
$$

- Then the covariance matrix $B$ for different investment programs of collaborative innovation can be calculated by using the expected return matrix.

$$
B=\left[\begin{array}{llllll}
84348 & 71073 & 69461 & 69069 & 68971 & 68953 \\
71073 & 59917 & 58562 & 58233 & 58150 & 58135 \\
69461 & 58562 & 57238 & 56916 & 56836 & 56821 \\
69069 & 58233 & 56916 & 56597 & 56517 & 56502 \\
68971 & 58150 & 56836 & 56517 & 56437 & 56422 \\
68953 & 58135 & 56821 & 56502 & 56422 & 56407
\end{array}\right] .
$$

The minimum risk and optimal weights combination under different expected earnings can be generated by using MATLAB's LMI Toolbox. The results are shown in Table 1.

The table shows us that in dynamic market the investment returns and risks change with the rate of return on innovation investment in different competitive context, and robustness is embodied in this changing process. The investment returns of collaborative innovation for enterprises can be optimized in the decision-making process. We can also get some relevant conclusions: 
Table 1: Portfolio results of robust optimization under different expected earnings

\begin{tabular}{lcllllll}
$E(\pi)$ & $\chi^{2} \times 10^{-8}$ & $w_{1}$ & $w_{2}$ & $w_{3}$ & $w_{4}$ & $w_{5}$ & $w_{6}$ \\
\hline 112.5 & 5.7732 & 0.1781 & 0.1657 & 0.1643 & 0.1640 & 0.1639 & 0.1639 \\
113 & 5.7730 & 0.1794 & 0.1655 & 0.1641 & 0.1637 & 0.1637 & 0.1636 \\
113.5 & 5.7733 & 0.1785 & 0.1654 & 0.1643 & 0.1640 & 0.1639 & 0.1639 \\
114 & 5.7728 & 0.1786 & 0.1657 & 0.1642 & 0.1639 & 0.1638 & 0.1638 \\
114.5 & 5.7730 & 0.1788 & 0.1657 & 0.1642 & 0.1638 & 0.1638 & 0.1637 \\
\hline
\end{tabular}

Firstly, $\chi^{2}$ varys according to the change of portfolio expected return $E(\pi)$. As we can see from Table 1, the risk of enterprise cooperative innovation is minimal when expected return is $(E(\pi)=114)$. It shows that the benefits and risks of cooperative innovation will coexist under dynamic environments. By controlling different collaborative innovation portfolio we can guide the enterprises making trade-off between risk and return of the investment decision-making and make effective investment decisions.

Secondly, under the dynamic environments the expected return and investment risk of an enterprises have no strict positive correlation. Through the analysis of the robust optimization we can calculate the minimum risk and optimal weights combination under different expected returns. Enterprises can develop the optimization investment weight $w_{i}$ for a variety of collaborative innovation scheme, thus providing science analysis to cope with innovation risk for enterprises.

Finally, the proposed robust optimization model of collaborative innovation under dynamic environments can reflect the uncertainty and investment risk that an individual enterprise faces in collaborative innovation. For partners of collaborative innovation, only if all participants accurately analyze the benefit and risk in the collaborative innovation, they can then effectively deal with market volatility and improve expected return of collaborative innovation investment.

\section{Discussions and conclusion}

In the dynamic environment, enterprises would be facing a certain risk of innovation and market in the process of collaborative innovation. Hence, the portfolio decision-making behaviors in the collaborative innovation are crucial for the enterprise. We apply the robust optimization method to analyze the enterprise's decision of the investment to the collaborative innovation. The result shows that in the dynamic environment the enterprise could achieve the investment goals with maximum investment return and minimum risk by controlling the proportion of the portfolio.

The assumption of this research actually is that the participating enterprises in the collaborative innovation have comparable power to determine the investment portfolio. For further research, it can be relaxed by considering one or more leading enterprises collaborative innovation. 


\section{acknowledgements}

This study is supported jointly by National Science Foundation of China for funding the projects (Grant No.71473067), the New Century excellent Talents in University of China (Grant No. NCET-12-1081), and the Research Center of Information Technology \& Economic and Social Development of Zhejiang Province for funding the project.

\section{References}

[1] J. Chen, Y. J. Yang, Theoretical basis and content for collaborative innovation, Studies in Science of Science 30 (2) (2012) 161-164.

[2] Y. X. Hong, Some conceptions on innovation-driven growth and collaborative innovation, Economic Theory and Business Management 5 (2013) 5-12.

[3] G. P. Zeng, Y. Z. Gou, L. Liu, From innovation system to innovation ecosystem, Studies in Science of Science 31 (2013) 4-11.

[4] Q. Zhou, D. M. Zeng, D. Zhu, Firm r\&d network-based game and investment decisions analysis, Journal of Industrial Engineering and Engineering Management 31 (2013) 4-11.

[5] Y. Qi, M. Zhang, G. Ding, Strategy on resource-sharing among collaborative innovation entities based on game theory, China Soft Science Magazine 1 (2013) 149-154.

[6] J. Z. Xu, W. F. Zhao, L. J. Wang, Analysis of the relationship among agents of collaborative innovation system in equipment manufacturing industry based on game theory, China Soft Science Magazine 7 (2014) $161-171$.

[7] Y. Liu, L. R. Jian, H. H. Zhao, Y. Lin, Profit distribution model of university-industry collaborative innovation value chain based on double efforts, R \& D Management 27 (2014) 24-34.

[8] B. Huang, H. Chen, W. Huang, Research on revenue sharing mechanism in collaborative innovation with venture capital fund of funds, Chinese Journal of Management Science 23 (2015) 66-75.

[9] J. Arsenyan, G. ykzkan, O. Feyziolu, Modeling collaboration formation with a game theory approach, Expert Systems with Applications 42 (4) (2015) 2073-2085.

[10] R. A. D'Aveni, R. Gunther, Hypercompetition: Managing the dynamics of strategic maneuvering, Free Press. New York, 1994.

[11] M. E. Porter, Competitive Strategy: Techniques for Analyzing Industries and Competitors, Free Press. New York, 1980.

[12] R. Drazin, A. H. V. de Ven, Alternative forms of fit in contingency theory, Administrative Science Quarterly 30 (1985) 514-539. 
[13] J. P. Choi, Cooperative r\&d with product market competition, International Journal of Industrial Organization 11 (1993) 553-571.

[14] Y. Katsoulacos, D. Ulph, Endogenous spillovers and the performance of research joint ventures, The Journal of Industrial Economics 4 (1998) 333-357.

[15] R. Narula, Cooperative Strategies and Alliances, Pergamon Press, 2002.

[16] F. Chen, J. G. Sui, The research on synergetic innovation and its evolution of emerging industries: a case of the new energy automobile industry, Science Research Management 36 (1) (2015) 26-33.

[17] Goyal, Joshi, Networks of collaboration in oligopoly, The Journal of Industrial Economics 43 (2003) 57-85.

[18] Bala, Goyal, Non-cooperative model of network formation, Econometrica 68 (2000) 1181-1231.

[19] Kranton, Minehart, A theory of buyer-seller networks, American Economic Review 91 (2001) 485-508.

[20] S. Goyal, J. L. Moraga-Gonzalez, R \&d networks, Rand Journal of Economics 32 (2001) 686-707.

[21] S. Goyal, J. L. MoragaGonzlez, A. Konovalov, Hybrid r\&d, Journal of the European Economic Association 6 (2008) 1309-1338.

[22] N. V. Sahinidis, Optimization under uncertainty: state of the art and opportunities, Computers and Chemical Engineering 28 (2004) 971-983.

[23] A. L. Soyster, Convex programming with set-inclusive constraints and applications to inexact linear programming, Operations Research 21 (1973) 1154-1157.

[24] J. M. Mulvey, R. J. Vanderbei, S. A. Zenios, Robust optimization of large-scale systems, Operations Research 43 (1995) 264-281.

[25] D. Bertsimas, M. Sim, The price of robustness, Operations Research 52 (2004) 35-53.

[26] O. L. V. Costa, J. B. R. D. Val, A convex programming approach to h2-control of markovian jump linear systems, International Journal of Control 66 (1997) 557-579.

[27] Y. Gao, X. Y. Huang, Y. Li, Lmi-based robust optimization model of loan portfolio, Journal of Northeastern University (Natural Science) 28 (2007) 137-140. 